\title{
Appareil
}

$20 \mid 2018$

Arts contemporains et patrimoine

\section{Le Grand Voile de Caroline Coppey, œuvre in situ}

Jean-Louis Déotte

\section{(2) OpenEdition}

Journals

Édition électronique

URL : http://journals.openedition.org/appareil/2179

DOI : 10.4000/appareil.2179

ISSN : 2101-0714

Éditeur

MSH Paris Nord

Référence électronique

Jean-Louis Déotte, "Le Grand Voile de Caroline Coppey, œuvre in situ », Appareil [En ligne], 20 | 2018, mis en ligne le 26 octobre 2018, consulté le 30 juillet 2020. URL : http://journals.openedition.org/ appareil/2179; DOI : https://doi.org/10.4000/appareil.2179

Ce document a été généré automatiquement le 30 juillet 2020.

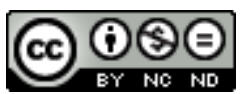

Appareil est mis à disposition selon les termes de la Licence Creative Commons Attribution - Pas d'Utilisation Commerciale - Pas de Modification 4.0 International. 


\title{
Le Grand Voile de Caroline Coppey, œuvre in situ
}

\author{
Jean-Louis Déotte
}

Figure 1. Caroline Coppey, Le Grand Voile, 2014

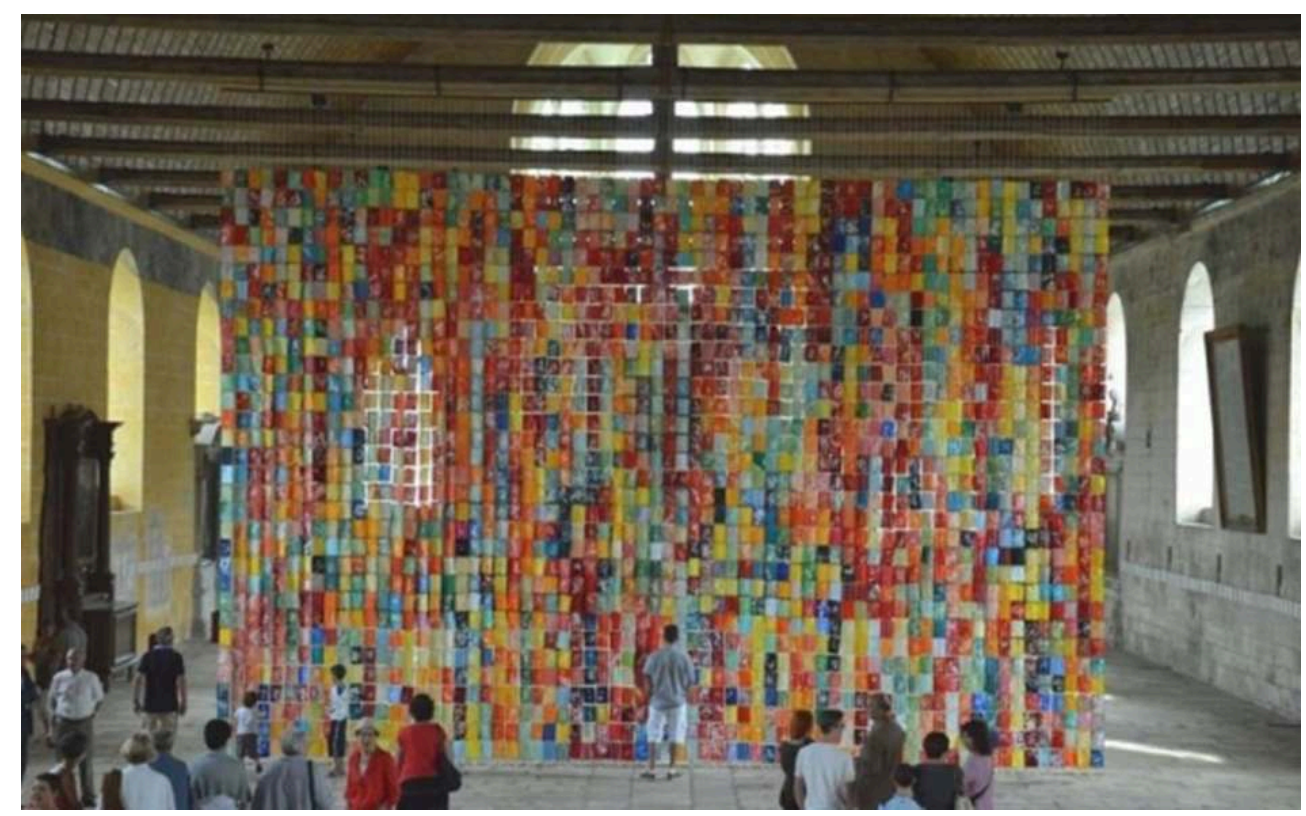

Source : Patricia Piquet pour L'Yonne républicaine

$1 \quad$ Il était dans la tradition des hôpitaux du Moyen Âge d'accueillir aussi bien les étrangers de passage que les malades. C'est cette double tradition que perpétue l'actuel directeur de l'hôpital, Frédéric Roussel. D'une part celle des soins au sens large, distribués gratuitement à une cité - c'est l'aspect care - ; de l'autre, celle de l'accueil de l'étranger. Ici, il s'agit en l'occurrence d'accueillir l'artiste Caroline Coppey, qui a appris son métier à l'Université de Strasbourg. D'autres "étrangers» devraient lui succéder dans les 
années à venir : Didier Guth avec le groupe franco-allemand Plakat, puis Germain Rœsz, Sylvie Villaume, Diane Watteau et Sandrine Morsillo.

2 On sait que l'hôpital de Tonnerre, fondé à la fin du XIII siècle par Marguerite de Bourgogne, aidée en cela par quelques compagnes appartenant à l'aristocratie, est la plus grande halle médiévale au monde, à mettre en rapport avec les Hospices de Beaune ${ }^{1}$. Sa destination a toujours été le care. Le mot care, très courant en anglais, est à la fois un verbe qui signifie "s'occuper de ", «faire attention", "prendre soin », « se soucier de » et un substantif qui pourrait, selon les contextes, être rendu en français par soins, attention, sollicitude.

Sinalité de la fondation est éminemment religieuse - une femme appartenant à la lignée royale, ayant reçu une éducation la destinant à exercer le pouvoir politique et qui, après son veuvage, décide de se mettre au service des pauvres, au milieu des plus démunis, en faisant vœu de pauvreté et de chasteté -, il n'en va plus de même depuis la laïcisation complète de l'hôpital et la professionnalisation de son personnel. Même si la philosophie du care appartient à l'éthique des relations intersubjectives, avec des auteurs anglo-saxons comme Francesca Cancian, Carol Gilligan, Joan Tronto, Jean Watson, en France l'inflexion a été plus sociale-politique (Sandra Laugier ${ }^{2}$, Pascale Molinier, Serge Guérin).

On peut faire la proposition suivante: dans des sociétés comme les nôtres où la politique de l'État-providence est manifestement dans une impasse parce que la redistribution des richesses ne s'effectue plus égalitairement, y compris dans les sociétés scandinaves, le temps est peut-être revenu d'un certain évergétisme ${ }^{3}$, qui ne serait plus le fait de citoyens fortunés (comme les mécènes anglo-saxons sur le modèle romain), mais d'institutions qui, dans des situations locales désastreuses, élargiraient le champ de leurs activités à la prise en charge de populations déshéritées, en particulier sur le plan psycho-social.

5 Bernard Stiegler pose ainsi le problème de l'auto-dévaluation collective actuelle de la nouvelle "plèbe" en termes de destruction du narcissisme primaire, distingué classiquement, à la suite de Freud, du narcissisme secondaire ${ }^{4}$. Quand la dépréciation de soi se généralise et s'extériorise, par exemple du fait de l'extension du chômage, de l'exclusion sociale, du rejet par le système scolaire, etc., et devient haine de soi, elle fait le lit du populisme. Sans narcissisme primaire inconscient, sans cette santé native de l'individu qui se sait socialement reconnu parce qu'il a une place dans le champ social, comment pourrait-il y avoir autre chose que cette désertion politique à laquelle nous assistons? Autre chose que ce mépris de la classe politique et par extension cette haine des élites? Cette souffrance de la nouvelle "plèbe » se manifeste régulièrement par la multiplication des émeutes urbaines ${ }^{5}$, ou des incendies d'automobiles dont seuls les journaux locaux font état.

6 Comment la nouvelle " plèbe " pourrait-elle « aimer » l'Europe alors que cette dernière n'a pour elle aucune consistance en termes d'hospitalité ? Encore faudrait-il voyager, parcourir ses confins. Si elle ne la parcourt pas, peut-être faut-il la faire venir chez elle? De la même manière, on peut être atterré par le niveau esthétique des constructions privées récentes dans de petites ou moyennes communes, où le maire uniquement peut donner son aval. Seules les institutions publiques, les commandes de l'État en premier lieu, peuvent et doivent actuellement faire imaginer qu'une autre architecture est possible, entre le sous-produit industriel et la copie du patrimoine ${ }^{6}$. 
On peut, par la possession de diplômes, sociologiquement appartenir à la prétendue «élite » et dans un même temps se sentir parfaitement étranger dans cette société dont les règles nous échappent.

La vérité, c'est qu'elles échappent à tous et en particulier aux experts en économie, dont la seule tâche est de faire oublier leurs erreurs passées.

Ce que nous avons tous en commun, c'est le fait de croire que les choses qui sont l'ont toujours été et le seront toujours. On peut appeler cela lâchement, comme le dernier Paul Veyne ${ }^{7}$, la " force des choses ». C'est là croire à l'éternelle répétition du Même, ce qu'un certain Nietzsche nommait l'« éternel retour». C'est ne plus avoir foi dans l'exception, c'est-à-dire dans le retournement du temps « comme un gant », comme le confirme le désespoir du dernier Benjamin ${ }^{8}$.

On doit pouvoir prendre soin de cette société en lui rendant le goût de l'exception. C'est l'étranger au sens large qui, en rendant le très familier, le trop répétitif, étrange permet de le découvrir dans sa singularité exceptionnelle. Notre société est en fait la chose la plus énigmatique qui soit, parce que toujours déjà-là : nous sommes venus au monde en son sein, aussi comment comprendre ce dont nous sommes les produits? Le paradoxe est qu'il est plus aisé de faire l'ethnographie d'une société exogène, d'emblée opaque, que la sociologie de notre tribu, immédiatement transparente.

Le Grand voile de Caroline Coppey, avec ses 2176 couleurs qui viennent rompre la continuité spatiale de la nef de la grande halle de Notre-Dame des Fontenilles, joue ce rôle. Il fallait en finir avec ce grand volume vide qui, depuis son désœuvrement comme salle d'accueil hospitalière, aurait pu finir comme marché, hangar à foin, lieu d'expositions commerciales, caserne, etc. Ces initiatives iconoclastes étaient devenues possibles, parce que l'extrême familiarité rendait l'architecture exceptionnelle invue et pour cette raison, inutile. Ce qui procède de l'habitude permet une grande économie d'énergie: on lance son chapeau sur la patère sans même la viser. Souvent l'art contemporain depuis Duchamp consiste, puisqu'on ne voit plus comme telles les œuvres d'art, à les rendre prosaïques, et inversement à rendre étranges les choses les plus prosaïques. Cette «étrangéisation » est le principe du théâtre épique de Brecht. Pour rendre compte du même mouvement, Kracauer utilise le terme de rédemption ${ }^{9}$ des choses par l'appareil photographique, puis cinématographique, ou de «sauvetage du réel ».

Bien sûr qu'un mur blanc aurait pu aussi réaliser cet office d'étrangéisation. Mais on voit bien dans l'abbatiale cistercienne de Pontigny, toute proche, que cela ne suffit pas. Ce voile doit être une parure, un apparat, un appareil décoratif au bon sens du terme. Il serait temps, en cette magnifique occasion, de restituer à la fonction décorative toute sa grandeur, toute sa nécessité, que reconnaissait implicitement Monet lui-même ${ }^{10}$. C'est le classicisme qui a séparé l'œuvre de son «hors-d'œuvre décoratif», l'œuvre « pure » de ce qui prépare la fonction de réception, rituelle ou esthétique. Or, peut-on penser séparer le temple de sa colonnade, la statue de ses vêtements, le tableau de son cadre ? Kant posait de telles questions dans son Esthétique ${ }^{11}$. Peut-on séparer, depuis le $\mathrm{XIX}^{\mathrm{e}}$ siècle, les œuvres dont on établit l'histoire et dont on célèbre la grandeur de cet appareil esthétique qu'est le musée ? Musée sur lequel se reporte donc la fonction de préparation de la réception esthétique, autrefois dévolue aux cadres des tableaux et autres supports de statues.

11 La décoration, qui suppose toujours un ordre, une géométrie, une logique, une répétition, donc peut-être une écriture au sens large, rend encore plus significative la 
chose qu'il faut distinguer. C'est la fonction qu'Oleg Grabar ${ }^{12}$ attribuait aux muqarnas de l'architecture musulmane. Les muqarnas sont des éléments décoratifs en forme de nids d'abeille et réalisés en stuc peint, en bois, en pierre ou en brique. Ces éléments dégringolent en stalactites ou garnissent les voûtes ou l'intérieur des coupoles de nombreux bâtiments musulmans.

Pour Grabar, ces éléments supposant une géométrie théorique très élaborée, dont les lois mathématiques sont synthétisées aujourd'hui au titre du "pavage du plan", constituaient des ensembles devant marquer la rupture entre l'espace profane et l'espace sacré. Bref, la décoration préparait la méditation et la prière dans une salle quasi vide, la mosquée, puisque seule l'orientation orientale était destinale.

Cependant, à la différence de cet ordre proprement cosmétique, au sens où il ne peut être séparé d'une référence théologique, l'esthétique moderne, en particulier à partir de Matisse ou de Simon Hantaï, est à la recherche de ses propres lois par l'introduction du hasard, obtenu par exemple par frottage des éléments. La proposition de Caroline Coppey s'inscrit dans cette histoire dont il faut maintenant reconstituer les étapes sur de nouvelles bases. À partir du moment où la frontière entre l'œuvre en soi et sa parure s'est effacée, le "décoratif » ne peut plus se contenter de répéter le Même. Il doit intégrer l'exceptionnalité de l'œuvre. Si c'est une écriture, c'est-à-dire un espacement réglé entre les éléments, alors ces derniers doivent être à chaque fois différents, voire uniques. C'est cette unicité de chaque couleur-élément que revendique Caroline Coppey. Selon elle, chaque couleur de sa palette n'est pas seulement différente de toutes les autres (ce serait rester dans le quantitatif de la définition de la couleur en termes de longueur d'onde), mais unique, c'est-à-dire non commutable, comme le sont les phonèmes ${ }^{13} \mathrm{~d}^{\prime}$ une langue donnée. Mais à la différence des phonèmes, les éléments de son Grand Voile ne sont pas en nombre fini. Dès lors sa tâche, son faire-œuvre, n'a pas de limites : elle consiste dans une répétition qui procède par creusement de l'élément lumineux, la chose la plus évidente pour les humains, seuls capables de voir les couleurs, qui devient ainsi la chose plus opaque. Il est évident alors que le lieu de cette expérimentation, la grande halle romane de Tonnerre, devient le lieu d'un double mouvement spéculatif : d'un côté quelque chose comme la constitution d'une langue visuelle universelle dont les éléments constitutifs seront innombrables; de l'autre, une revendication de l'Un.

\section{BIBLIOGRAPHIE}

Benjamin Walter, Sur le concept d'histoire, «thèse VIII », in Écrits français, Paris, Gallimard, coll. « Folio essais », [1940] 1991.

Coppey Caroline, Claude Monet : à l'école de l'œil, Paris, L'Harmattan, 2013.

Francastel Pierre, L'Impressionnisme, Paris, Denoël/Gonthier, 1974.

Grabar Oleg, Penser l'art islamique. Une esthétique de l'ornement, conférences IMA, Paris, Albin

Michel, 1992. 
Kant Emmanuel, Critique de la faculté de juger ou Critique du jugement, A. Philonenko (trad.), Paris, Vrin, 1965.

Kracauer Siegfried, Théorie du film. La rédemption de la réalité matérielle, traduction de Daniel Blanchard et Claude Orsoni, Paris, Flammarion, 2010.

Le Clech-Charton Sylvie, L'Hôtel-Dieu de Tonnerre. Métamorphose d'un patrimoine hospitalier, $\mathrm{XIII}^{e}-\mathrm{XX} \mathrm{X}^{e}$ siècle, Langres, éditions Guéniot, 2012.

Molinier Pascale, Laugier Sandra et Paperman Patricia, Qu'est-ce que le care ? Souci des autres, sensibilité, responsabilité, Paris, Petite Bibliothèque Payot, 2009.

Stiegler Bernard, Aimer, s'aimer, nous aimer. Du 11 septembre au 21 avril, Paris, Galilée, 2003.

Veyne Paul, Le Pain et le cirque. Sociologie historique d'un pluralisme politique, Paris, Le Seuil, 1976.

\section{NOTES}

1. Le Clech-Charton Sylvie, L'Hôtel-Dieu de Tonnerre, Langres, Éditions Dominique Guéniot, 2012.

2. Sandra Laugier et la notion de "politique de l'ordinaire». Voir Molinier Pascale, Sandra Laugier, Patricia Paperman, Qu'est-ce que le care? Souci des autres, sensibilité, responsabilité, Paris, Petite Bibliothèque Payot, 2009.

3. Au sens de l'analyse de Paul Veyne, Le Pain et le cirque. Sociologie historique d'un pluralisme politique, Paris, Le Seuil, 1976.

4. Stiegler Bernard, Aimer, s'aimer, nous aimer. Du 11 septembre au 21 avril, Paris, Galilée, 2003.

5. Un laboratoire de sociologie dirigé par Alain Bertho a constitué, dans le cadre de la Maison des Sciences de l'Homme Paris Nord, un Observatoire des mobilisations visuelles en 2013.

6. Pourquoi fallait-il que la nouvelle médiathèque de Tonnerre, dite Cœurderoy, en hommage dérisoire à ce grand anarchiste, soit un mauvais compromis entre un hangar agricole et la toiture de la grande halle de l'Hôtel-Dieu? Les édiles tonnerroises auraient dû se souvenir de son «Hourrah ou la révolution par les cosaques... ».

7. À propos de la diffusion irréversible du christianisme à partir de Constantin.

8. Celui des thèses Sur le concept d'histoire, en particulier la « thèse VIII ».

9. Kracauer Siegfried, Théorie du film. La rédemption de la réalité matérielle, traduction de Daniel Blanchard et Claude Orsoni, Paris, Flammarion, 2010.

10. Francastel Pierre, L'Impressionnisme, Paris, Denoël Gonthier, 1974, à propos des « Nymphéas ». Cité par Caroline Coppey, Claude Monet : à l'école de l'œil, Paris, L'Harmattan, 2013.

11. Kant Emmanuel, Critique de la faculté de juger ou Critique du jugement, A. Philonenko (trad.), Paris, Vrin, 1965.

12. Grabar Oleg, Penser l'art islamique. Une esthétique de l'ornement, conférences IMA, Paris, Albin Michel, 1992.

13. Phonème, selon le dictionnaire Wikipédia : «En phonologie, domaine de la linguistique, un phonème est la plus petite unité discrète ou distinctive (c'est-à-dire permettant de distinguer des mots les uns des autres) que l'on puisse isoler par segmentation dans la chaîne parlée. Un phonème est en réalité une entité abstraite, qui peut correspondre à plusieurs sons. Il est en effet susceptible d'être prononcé de façon différente selon les locuteurs ou selon sa position et son environnement au sein du mot (voir allophone). Les phones sont d'ailleurs les différentes réalisations d'un phonème. Par exemple $[\mathrm{b}]$ dans croc [kظo $]$, et $[\mathrm{b}]$ dans gros [gьо] sont deux phones différents du même phonème $/ \mathrm{b} /$. On transcrit traditionnellement les phonèmes par des lettres placées entre des barres obliques: $/ \mathrm{a} / \mathrm{/} / \mathrm{t} / \mathrm{/} / \mathrm{H} /$, etc., selon la règle un phonème $=$ un symbole.» 


\section{RÉSUMÉS}

À partir du Grand Voile de Caroline Coppey, installé de manière éphémère en 2014 à l'Hôtel-Dieu de Tonnerre, l'article développe des réflexions successivement éthiques, esthétiques et métaphysiques pour penser les différents rapports d'une œuvre à l'espace qu'elle vient occuper : espace muséal, espace hospitalier, espace de dévoilement. Dans ce texte de 2015, l'auteur parle encore d'œuvre in situ. C'est tout particulièrement sur l'œuvre décorative et son langage que l'accent est porté.

\section{INDEX}

Mots-clés : Hospitalité, espace, étrangéisation, décoration

\section{AUTEUR}

\section{JEAN-LOUIS DÉOTTE}

Jean-Louis Déotte a été professeur de philosophie à l'Université Paris VIII, Saint-Denis, coordinateur du thème « Esthétique, arts et industries » à la MSH Paris Nord, directeur de la collection « Esthétiques » chez L'Harmattan et enfin, directeur de la revue Appareil. 